\title{
The Selectional Constraint of the Chinese Progressive Operator zai
}

\author{
Zhang Mengjie ${ }^{1}$, Zhu Haiting ${ }^{2}$, Lin Yunqing ${ }^{3, *}$ \\ ${ }^{1}$ School of Foreign Languages, Beihang University, Beijing, China \\ ${ }^{2}$ School of Foreign Languages, Minzu University of China, Beijing, China \\ ${ }^{3}$ English Department, Beijing International Studies University, Beijing, China
}

Email address:

by1612102@buaa.edu.cn (Zhang Mengjie), zht198663@163.com (Zhu Haiting), ylin@bisu.edu.cn (Lin Yunqing)

${ }^{*}$ Corresponding author

\section{To cite this article:}

Zhang Mengjie, Zhu Haiting, Lin Yunqing. The Selectional Constraint of the Chinese Progressive Operator zai. International Journal of Language and Linguistics. Vol. 9, No. 3, 2021, pp. 106-110. doi: 10.11648/j.ijl1.20210903.17

Received: April 15, 2021; Accepted: May 4, 2021; Published: June 1, 2021

\begin{abstract}
Previous studies on the Chinese progressive mainly focus on the distinctions between Chinese progressive zai and the continuous $-z h e$, but few has noticed that the progressive zai is sensitive to the plurality of the event encoded by the predicate in its scope. Inspired by the observations of the selectional constraints of the English progressive, this paper aims to find out the selectional constraints of Chinese progressive zai. It is argued that Chinese progressive zai requires its modified predicates to denote singular durative events as its English counterpart do. Given that no studies have illustrated the semantic criterion for identifying predicates denoting singular events in Mandarin, this study proposes that singular telic (incremental) predicates compatible with zai are those with singular atoms or singular groups as the incremental themes and singular atelic predicates co-occurring with zai are those being incremental homogeneous. An incremental accomplishment with a singular atom as the theme is expressed by the combination of an incremental verb and a quantized incremental object with the cardinal numeral quantifier $y i$ 'one' as the measure phrase, while that with a singular group as the theme is expressed by the combination of an incremental verb and a definite object modified by the definite determiner like $n a$ 'that/those'. In contrast, incremental homogeneous predicates are expressed by activities or plural achievements.
\end{abstract}

Keywords: Progressive Operator zai, Telicity, Atelicity, Singularity, Plurality

\section{Introduction}

Schedule English progressives are sensitive to different kinds of predicates they are co-occurring with [1,2]. "A telic predicate that is generated by means of (i) a quantized incremental theme argument (ii) with an overt measure phrase and/ or determiner quantifier, is unacceptable in a sentence with progressive interpretation, or it reduces the range of its interpretive possibilities" [2]. For instance,

(a) ? John was eating three pears when I arrived. ${ }^{1}$

The predicate 'eat three pears' sounds unnatural when it is modified by the progressive operator in the example (a), because it is a telic predicate generated by the combination of

1 The question mark here is to show the degree of unacceptance or unnaturalness of the example. The more question markers, the more unacceptable/ unnatural the example is. an incremental-theme verb 'eat' and the quantized incremental theme 'three pears' and the latter is modified by the overt measure phrase 'three'.

Similar to its English counterpart, the Chinese progressive operators zai has some selectional constraint as well. It is also incompatible with accomplishments with quantized incremental themes.

In a line with its English counterpart, the Chinese telic predicate chi liuge pingguo 'to eat six apples' is an incremental accomplishment composed by the incremental-theme verb chi 'to eat' and the quantized incremental theme liuge pingguo 'six apples' with the cardinal numeral quantifier liu 'six' as the measure phrase (see [3, 4]). That incremental accomplish is infeasible to co-occur with the progressive operator zai in the example (b). 
(b) ?? Zhangsan zai chi liu ge pingguo

Zhangsan PRO eat six CL apple ${ }^{2}$

?Zhangsan is eating six apples.

The reason why the incompatibility arises might be that intuitively one can eat only one apple rather than six apples at a time. If the event of eating one apple is a singular one and the event of eating six apples is a plural one obtained by summing together six one-apple-eating events, it is expected that the progressive zai modifies only predicates denoting singular events $^{3}$. This is actually what Ferreira [5] argues for the semantics of English progressives. According to Ferreira [5], progressives are about singular events. The predicate denoting a singular event is good enough to appear in a progressive sentence while the predicate denoting a plural event is out in a progressive sentence. Following this line of thought, the example (c) is grammatical because the involved predicate denotes a singular one-apple-eating event.

(c) Zhangsan zai chi yi ge pingguo

Zhangsan PRO eat one CL apple

Zhangsan is eating an apple.

What is interesting is that the example (b) becomes felicitous when the incremental theme is further modified by the demonstrative $n a$ 'those', as in the example (d).

(d) Zhangsan zai chi zuozi shang de na liu ge pingguo

Zhangsan PRO eat table up $\mathrm{DE}^{4}$ that six CL apples

Zhangsan is eating those six apples on the table.

Moreover, contrary to Ferreira's [5] observation, predicates denoting apparent plural events can appear in progressive sentences as well. For example,

(e) Keren men zai yi ge yi ge de jin wu

Visitor PL PRO one CL one CL DE enter room

Visitors were entering into the room one by one.

The event of visitors' arriving in the denotation of the example (e) is a plural one consists a series of singular visitor's arriving events, which is expected to be incompatible with zai. But it turns out that the predicate is felicitous to appear in the example (e).

On the one hand, this study argues that Ferreira [5] is basically right in observing that progressives are about singular events. But his general focus on the distinction between progressives and imperfectives leaves him no room to give an explication of how to define singular verbal predicates. This leads to the result that his account cannot explain the well-formedness the example (d) and the example (e). On the other, previous studies on Chinese progressives mostly concentrate on the difference between the dynamicity of zai and durativity of -zhe [6-7]. Few has reported and explained the sensitivity of zai to its co-occurring predicates. In light of these research gaps, the aims of this study are twofold. Firstly, this study proposes that the selectional constraint of Chinese progressive operator zai is that it

2 PRO is abbreviated for progressive and CL classifier.

3 Here the event refers to a durative event. Progressive are incompatible with punctual predicates. Without further specification, singular telic and atelic events in this study are all durative events.

$4 \mathrm{De}$ is the abbreviation for marker of adjectival or adverbial modification. It represents adverbial modification in both examples of (d) and (e). requires the modified predicate to be singular. Secondly, this study illustrates the criteria for identifying singular telic and atelic predicates in Mandarin Chinese. It is found that Chinese singular telic (incremental) predicates are those which have singular atoms or singular groups as the incremental themes and Chinese singular atelic predicates are those which are incremental homogeneous.

\section{Singular Telic and Atelic Predicates in Mandarin}

Bach [8] takes atelic predicates to be verbal counterparts of mass nouns, and telic predicates to be the verbal counterparts of count nouns. It follows that atelic events are uncountable and telic events are countable. However, all atelic events are potentially countable when they develop into some contextually maximal event. For instance, the event of John's running is an atelic one. But it can be shifted into a telic one when the atelic predicate 'John ran' is modified by the quantitation expression like 'three times'. Therefore, Rothstein [9] argues that verbal predicates always have their denotation in the count domain Although both telic and atelic predicates are countable, they are counted in different ways. In Rothstein's [9] analysis, telicity is characterized by atomicity. Since telic predicates are individuated and atomic, we can count telic events directly just as what we do in the case of atomic concrete objects in the denotation of count nouns. In contrast, atelic predicates have no atomic structure, and hence cannot be counted in the same way as telic predicates are counted. Then the question at issue is how to identify singular event in the count denotations of Chinese telic and atelic predicates. Section 2.1 introduces how to identify singular telic predicate in Chinese and section 2.2 illustrates the conditions for atelic predicates to denote one and the same singular events. During the process of identifying singular telic and atelic predicates, the selectional constraint of zai is illustrated.

\subsection{Singular Telic Predicates in Mandarin}

To know how telic predicates are counted, we firstly need to clarify the notion of telicity. This study follows Krifka's [10] definition of telicity as quantization.

$$
\mathrm{P} \text { is quantized: } \mathrm{Q}(\mathrm{P}) \leftrightarrow \forall \mathrm{x} \forall \mathrm{y}[(\mathrm{P}(\mathrm{x}) \wedge \mathrm{P}(\mathrm{y}) \rightarrow \neg(\mathrm{x} \sqsubset \mathrm{y})]
$$

Formula (1) says that if a predicate $\mathrm{P}$ is telic, then for all $\mathrm{x}$ and $\mathrm{y}$ in the denotation of $\mathrm{P}, \mathrm{x}$ cannot be a part of $\mathrm{y}$. In other words, if $\mathrm{P}$ is telic, there is no part of the denotation of $\mathrm{P}$ is also in the denotation of $\mathrm{P}$. According to this definition, the predicates chi liuge pingguo 'to eat six apples' in the example (b) and chi zuozishang na liuge pingguo 'to eat those six apples on the table' in the example (d) are both telic, because no part of the event of eating six apples is itself an event of eating six apples and no part of the event of eating those six apples is itself an event of eating those six apples.

If the hypothesis that zai selects only singular predicates is on the right track, the different behaviors of the predicates in the examples (b) and (d) suggest that chi liuge pingguo 'to eat 
six apples' denotes a set of plural telic events while chi zuosi shang na liuge pingguo 'to eat those six apples on the table' denotes a set of singular telic events. Why is it the case that the event in the denotation of the example (b) is a plural one while that in the denotation of the example (d) is a singular one? Before answering this question, we firstly have a look at how Rothstein [9] illustrate the event encoded by the predicate 'ate two apples' in the example (f).

(f) John ate two apples.

On her analysis, 'ate two apples' can denote either a singular atomic two-apple eating event involving as the theme a group of two apples or a plural event consisting of two atomic one-apple-eating events. Adapting and modifying the group atom formation and plural operation * in Link [11], the semantics of the predicate 'ate two apples' is formulized in Formula (2).

$$
\begin{gathered}
{[[\text { ate two }} \\
\text { apples }]]= \\
\lambda e \exists x \text { Atom }_{\text {group }}(x) \wedge \text { apple }(x) \wedge \quad \text { theme }(e, x) \wedge \text { eat }(e) \\
\wedge \exists y[* \operatorname{apple}(y) \wedge|y|=2]] \text { or } \\
{[[\text { ate two apples }]]=\lambda e \exists x[* \operatorname{eat}(e) \wedge \quad * \text { theme }(e, x) \wedge \quad *} \\
\operatorname{apple}(x) \wedge|y|=2]
\end{gathered}
$$

The Chinese predicate chi liangge pingguo 'to eat two apples' is expected to denote either a singular event of eating two apples or a plural event composed by two one-apple-eating events, if it follows the suit of its English counterpart. Concerning the infelicitous usage of zai in the example (g), we assume that the denoted event can only be a plural one instead of a singular one.

\section{(g) ?? Zhangsan zai chi liang ge pingguo ${ }^{5}$}

Zhangsan PRO eat two CL apple

?Zhangsan is eating two apples.

What follows will show that our assumption is right. The plurality of the numeral-classifier accomplishment can also be supported by the negation of non-culminating accomplishment in Mandarin. Consider the scenario in the example (h).

(h) Zhangsan chi le liang ge pingguo, keshi dou mei chi wan Zhangsan eat PER ${ }^{6}$ two CL apple but DIS not eat up *Zhangsan ate two apples, but he finished none of them.

A group of scholars have observed that the distributive operator dou 'all' ${ }^{7}$ is obligatory to appear in the negating clause for Chinese non-culminating accomplishment when the number in the numeral-classifier object is more than one [12-14]. The obligatory presence of dou 'all' suggests that the two patients must be both involved in the eating event. That is, both apples are affected in the eating event.

\footnotetext{
5 Although not being ungrammatical, the example $(\mathrm{g})$ is at least unnatural. In mandarin, the more natural way to express the progression of events as such would be a sentence without numerals, like Zhangsan zai chi pingguo 'Zhangsan was eating the (two) apples'.

6 PER stands for the perfective operator and DIS the distributive operator.

7 A full exploration of the controversy nature of dou is beyond the scope of this paper. This study adopts the Lin's [15] view to take dou as a distributive operator.
}

Why the participants' involvement is relevant for our discussion of identifying singular incremental accomplishments? Because if the predicate chi liangge pingguo 'to eat two apples' is singular, it must have a group of two apples as the incremental theme. One of Landman's [16] arguments for the group formation is participants' involvement in a singular one event, as shown in the contrast between the example (i) and the example (j).

(i) David, Chris, Jerry and Tina gave a concert in Holland.

(j) The Talking Heads gave a concert in Holland.

Suppose that David, Chris, Jerry and Tina are the members of the Talking Heads group. In a scenario in which not all the members of the Talking heads are involved in giving a concert, the example (j) rather than the example (i) is true. So partial involvement is only possible in events with groups as participants.

Since both apples are necessarily affected in the example (h), the numeral-classifier argument liangge pingguo 'two apples' is impossible to function as the group theme of the predicate in the example $(\mathrm{g})$. The predicate chi liangge pingguo 'to eat two apples', different from its English counterpart being a singular atomic predicate, is a plural predication to sums of two apples. The event structure of the predicate chi liangge pingguo 'to eat two apples' is shown in Figure 1.

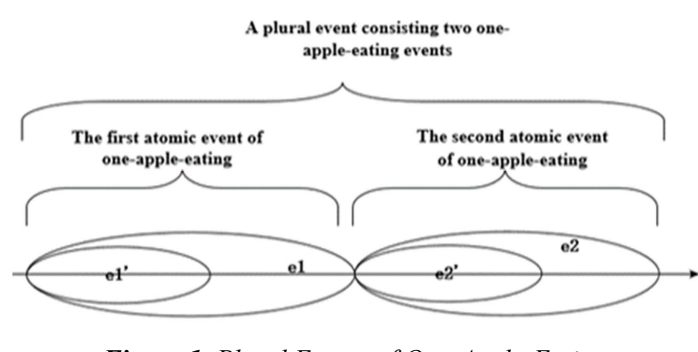

Figure 1. Plural Events of One-Apple-Eating.

Assuming that noun roots denote kind in Chinese and that individuating classifiers introduce atomicity [17-18], we give the semantics of the predicate chi liangge pingguo 'to eat two apples' in Formula (3).

$$
\begin{array}{r}
\text { pingguo }]]=\lambda e \exists x\left[* \operatorname{Atom}_{\text {natural liangge }}\left(\operatorname{apple}_{k}, x\right) \wedge \operatorname{count}(x)=\right. \\
2 \wedge * \operatorname{eat}(e) \wedge * \operatorname{theme}(e, x)]
\end{array}
$$

In contrast, when the demonstrative predicate chi na liangge pingguo 'to eat those two apples' occurs in a non-culminating sentence, those two apples are not necessarily both affected.

(k) Zhangsan chi le zuozi shang na liang ge pingguo,

Zhangsan ate PER table on that two CL apple

keshi mei chi wan

but not eat up

*Zhangsan ate those two apples, but did not finish.

In light of the fact that the example $(\mathrm{k})$ is true in a scenario where only part of one of those two apples are affected in the eating event, it is argued that those two apples function as a group to participate in the eating event. Hence the example (k) encodes a singular atomic two-apple-eating event involving as 
the theme a group of two apples. The event structure is shown in Figure 2.

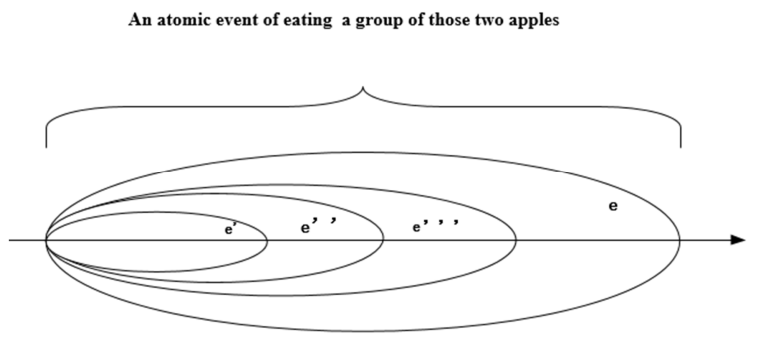

Figure 2. Singular Event of Two-Apple-Eating.

The semantics of chi na liangge pingguo 'to eat those two apples' is shown in Formula (4).

$$
\begin{gathered}
\begin{array}{c}
{[[\text { chi na liangge }} \\
\text { pingguo }]]=
\end{array} \\
\lambda e \exists x\left[\text { Atom }_{\text {group }(x) \wedge \operatorname{apple}_{k}(x) \wedge e a t(e) \wedge \text { theme }(e, x)}\right. \\
\wedge \exists y\left[\operatorname{Atom}_{\text {natural } \left.\left.(y)\left(\text { apple }_{k}, y\right) \wedge \quad|y|=2\right]\right]}(4)\right.
\end{gathered}
$$

Since the predicate in the example (g) denotes a plural event consisting of two atomic one-apple-eating events, it cannot co-occur with the progressive operator zai. In contrast, the predicate in the example (1) denotes an atomic two-apple eating event involving as the theme a group of two apples, it has no difficulty in appearing in a progressive sentence.

(1) Zhangsan zai chi na liang ge pingguo

Zhangsan PRO eat that two CL apples

Zhangsan is eating those two apples.

Therefore, the contrasts between the example (b) and he example (d), and also between the example (g) and the example (1), is explained. For a Chinese telic incremental accomplishment to be singular, its incremental theme must be modified by cardinal quantifiers not more than one or be modified by demonstratives. Only in this way, the incremental accomplishment has a singular atom or a singular group as the theme and denotes singular event.

\subsection{Singular Atelic Predicates in Mandarin}

Still, for the singular atelic predicates, the first thing needs to be clarified is the notion of atelicity. We again follow Krifka's [10] assumption to characterize atelicity as cumulativity, as shown in Formula (5).

$\mathrm{P}$ is cumulative: $\mathrm{CUM}(\mathrm{P}) \leftrightarrow \forall \mathrm{x} \forall \mathrm{y}[(\mathrm{P}(\mathrm{x}) \wedge \mathrm{P}(\mathrm{y})) \rightarrow \mathrm{P}(\mathrm{x} U \mathrm{y})]$

Formula (5) says that if $\mathrm{P}$ is cumulative, then for any $\mathrm{x}$ and $\mathrm{y}$ in $\mathrm{P}$, the sum of $\mathrm{x}$ and $\mathrm{y}$ is also in P. Consider the example (e) again. The sum of two events of visitors' entering into the room is still an event of visitor's entering the room. So the predicate keren men jinwu 'visitors to enter the room' is cumulative, and hence atelic ${ }^{8}$.

8 A singular event of a visitor's arriving is a punctual one, and it is contradictory to the durative meaning of the progressive zai. But the plural event of visitors' arriving is a plural one that is obtained by summing together repetitive arriving events. It is durative and atelic, and hence can co-occur with zai.
The next question is why the atelic event consisting of a series of subevents is still a singular one. Atelic events are theoretically unbounded in temporal extension and they cannot be individuated. Because a singular atelic event can be temporally extended to a bigger singular one, it is a singular atelic event as long as its different temporal extensions can be identified as one and the same atelic event. It follows that the question of identifying a singular atelic event turns out to be the issue of recognizing one and the same atelic event. This study employs the notion of incremental homogeneity, which is originally proposed to account for the felicitous usage of for-adverbials in Landman \& Rothstein [19], to track one and the same singular atelic event.

Incremental homogeneity [19]

Let VP be an eventive predicate, interpreted as event type $\alpha$ and $\mathrm{V}$ be the verbal head of VP, interpreted as event type V $\alpha$.

e in $\alpha$ is incrementally homogeneous with respect to $\alpha$ and $\mathrm{V} \alpha$ iff:

If $\tau(e, w)$ is defined then for every interval i such that $\tau\left(\mathrm{O}\left(\mathrm{e}, \mathrm{V}_{\alpha}\right)\right) \preccurlyeq \mathrm{i} \prec \tau(\mathrm{e}, \mathrm{w})$, then there is an event $\mathrm{e}^{\prime} \in$ $\alpha$ such that $\tau\left(\mathrm{e}^{\prime}, \mathrm{w}\right)=\mathrm{i}$ and $\mathrm{e}^{\prime} \sim \mathrm{e}$

"As an example, if e is an event of waltzing, then onset event $\mathrm{O}$ (e, WALTZ) is the smallest initial event in $\tau(\mathrm{e})$ that is big enough to count both as waltzing and as cross-temporally identical to $\mathrm{e}$ " [19]. If the onset event and $\mathrm{e}$ are cross-temporally identical, they can be counted as one and the same event for the counting purpose. The predicate waltz is incrementally homogenous because "if e is a waltzing event realized in $w$, we find, for each proper subinterval of $\tau(e, w)$ incrementally extending the running time of the waltzing-onset of e, an event cross-temporally identical to e, which is itself a waltzing event" [19]. Events incrementally extending the onset event are those which share the same beginning of the onset event.

Taking all of these into consideration, we claim that the atelic event $\mathrm{e}$ in the denotation of the predicate keren men jinwu 'visitors to enter into the room' in the example (e), although being seemingly a plural event, is actually a singular atelic event, because it is incremental homogeneous. The event of visitor's arriving the room has an onset event of the first visitor's entering into the room that is temporally identical to e and for any proper subinterval of the incremental extension of the onset event, we can find a visitors' arriving event that is cross-temporally identical to e. Its event structure is explicated in Figure 3.

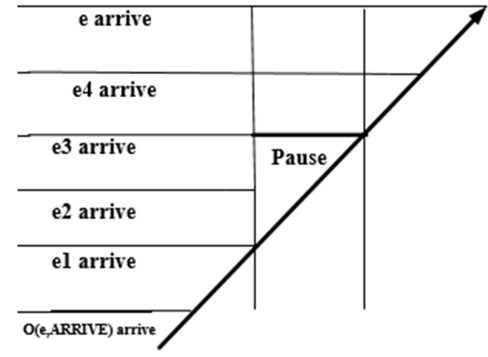

Figure 3. A singular atelic event consisting of iterative subevents of visitors' arriving. 
The predicate jinwu 'to enter into the room' is an achievement and the set of events in its denotation is instantaneous. The onset of the event in the denotation of the predicate keren men jinwu 'visitors to enter into the room' is the smallest event satisfying the predicate. Because the plural noun keren men 'visitors' also has singular visitor in its denotation [15], the onset event is the first visitor's arriving event as shown in the bottom line in Figure 3. Then any cross-temporally identical development of the onset event, that is, any event that shares the same beginning with onset event and incrementally extends the onset event, is in the denotation of the predicate. For example, e3, the first three visitors' entering the room, shares the same beginning with the onset event and temporally extends the onset event. So it is in the denotation of the predicate keren men jinwu 'visitors to enter into the room'. It follows that we can count all the events, e1, e2, e $3 \ldots$ and finally e, as one and the same event in Figure 3. Since the predicate keren men jinwu 'visitors to enter into the room' is singular, it is compatible with the progressive operator zai. This is the reason why the example (e) is felicitous. The semantics of keren men jinwu 'visitors to enter into the room' is given in Formula (5).

$$
\begin{array}{r}
{[[\text { keren men }} \\
\text { jinwu] }]=\exists e^{\prime}[* \\
\lambda e \exists y \exists x\left[* \operatorname{arrive}(e) \wedge \operatorname{theme}\left(e^{\prime} x\right) \wedge \operatorname{room}(e) \wedge\right. \\
\text { agent } \left.(e, x) \wedge * \operatorname{visitor}(x)]\left(e^{\prime}\right)\right] \quad(7)
\end{array}
$$

In addition to plural achievement, incremental homogenous predicates can also be expressed by activities in Mandarin. Because activities are incremental homogeneous, they are singular predicates felicitous to co-occur with progressive zai, as shown in the following example.

(m) Zhangsan zai paobu

Zhangsan PRO run

Zhangsan is running.

\section{Conclusion}

This study makes a tentative analysis of the selectional constraint of Chinese progressive operator zai. It is argued that zai modifies only singular verbal predicate. This study further illuminates how to identify singular telic predicate and atelic predicate in Mandarin Chinese. Due to the limited space, with regard to the telic predicates, this study only examines incremental accomplishments in Chinese. It is found that a Chinese telic incremental predicate is singular when it has as the incremental theme a singular atom or a singular group, while a Chinese atelic predicate is singular when it is incremental homogeneous.

\section{Acknowledgements}

This study was funded by China's Educational Department Social Science Foundation for Young Scholars' project “A distributional lattice approach to English syntactic categories acquisition" (18YJC740154) and the Academic Excellence Foundation of BUAA for PhD Students.

\section{References}

[1] Zucchi, S. (1999) Incomplete Events, Intensionality and Imperfective Aspect. Natural Language Semantics, 7 (2), 179 215.

[2] Filip, H. "The Telicity Parameter Revisited," In Proceedings of Semantic and Linguistic Theory XIV. Watanabe, K. \& Young, R. B. Eds. Ithaca, NY: CLC Publications. 2005.

[3] Yang, S.-Y. (2011) The Parameter of Temporal Endpoint and the Basic Function of -le. Journal of East Asian Linguistics, 20 (4), 383-415.

[4] Yang, J. and Wu, Y. (2015) Revisiting the (Non-) Existence of Accomplishments in Chinese. Modern Foreign Languages, 38 (6), 731-741 (in Chinese).

[5] Ferreira, M. (2016) The Semantic Ingredients of Imperfectivity in Progressive, Habituals, and Counterfactuals. Natural language semantics, 24 (4), 353-397.

[6] Smith, C. S. The Parameter of Aspect. Dordrecht: Kluwer Academic Publishers, 1997.

[7] Xiao, R. Z., \& McEnery, A. M. Aspect in Mandarin Chinese: A Corpus-based Study. Amsterdam/ Philadelphia: John Benjamins, 2004.

[8] Bach, E. (1986) The Algebra of Events. Linguistics and Philosophy, 9 (1), 5-16.

[9] Rothstein, S. Structuring Events: A Study in the Semantics of Lexical Aspect. Oxford: Blackwell, 2004.

[10] Krifka, M. "The Origins of Telicity", in Events and Grammar, S. Rothstein, Eds. Dordrecht: Kluwer, 1998.

[11] Link, G. "The logical Analysis of plurals and mass terms: A lattice-Theoretical Approach", in Formal Semantics-the Essential Readings, Portner, P. \& Partee, B. H. Eds. Blackwell, 1983.

[12] Li, X \& Duan. W. A Reanalysis of the Telicity of Chinese accomplishment. (forthcoming).

[13] Zhang, M. and Lin, Y. (2019) On Non-culminating Accomplishments in Mandarin Chinese. Modern Foreign Languages, 42 (5), 585-596 (in Chinese).

[14] Zhang, A. (2020) Referentiality, Individuation and Incompletive Readings. Journal of East Asian Linguistics, 29: 435-468.

[15] Lin, J.-W. (1998) Distributivity in Chinese and its Implications. Natural language semantics, 6 (2), 201-243.

[16] Landman, F. (1989) Groups, I. Linguistics and Philosophy, 12 (5), 559-605.

[17] Li, X. Numeral Classifiers in Chinese: The Syntax-Semantics Interface. Germany: Walter de Gruyter, 2013.

[18] Chierchia, G. (1998) Reference to Kinds across Language. Natural Language Semantics, (4), 339-405.

[19] Landman, F and Rothstein, S. (2012) The Felicity of Aspectual for-Phrase Part 2: Incremental Homogeneity. Language and Linguistics Compass, (2): 85-96. 